\title{
Limit cycles with imperfect valves: Implications for controllability of processes with large gains
}

\author{
Antonio Araújo and Sigurd Skogestad* \\ Department of Chemical Engineering, \\ Norwegian University of Science and Technology (NTNU), \\ Trondheim, Norway
}

Revision 3 (hopefully final) to Industrial \& Engineering Chemistry Research

October 5, 2006

\begin{abstract}
There is some disagreement in the literature on whether or not large plant gains are a problem when it comes to input-output controllability. In this paper, controllability requirements are derived for two kinds of input errors, namely restriced (low) input resolution (e.g. caused by a sticky valve) and input disturbances. In both cases, the controllability is limited if the plant gain is large at high frequencies. Limited input resolution causes limit cycle behavior (oscillations) similar to that found with relay feedback. The magnitude of the output variations depends on the plant gain at high frequency, but is independent of the controller tuning. Provided frequent input (valve) movements are acceptable, one may reduce the output magnitude by forcing the system to oscillate at a higher frequency, for example by introducing a faster local feedback (e.g. a valve positioner) or by pulse modulating the input signal.

Keywords: High gain, input disturbance, valve resolution, quantizer, limit cycle, controllability, PI-controller.
\end{abstract}

\section{Introduction}

The main goal of feedback control is to the keep the plant outputs $y$ within specifications in spite of disturbances, errors and uncertainty. A fundamental question arises: Is the process input-output controllable? There are many factors that need to be considered and one of them is the magnitude of the process gain. The gain depends on the frequency and, for multivariable plants, also on the input direction. To quantify this, the singular values $\sigma_{i}(G(j \omega))$ of the process transfer function $G(s)$ are considered. Of particular interest are the maximum and minimum singular values, denoted $\bar{\sigma}(G)$ and $\underline{\sigma}(G)$, respectively. In this paper, for simplicity, mainly SISO systems are considered, where $\bar{\sigma}(G(j \omega))=\underline{\sigma}(G(j \omega))=|G(j \omega)|$.

* Author to whom correspondences should be addressed: Department of Chemical Engineering, Sem Saelandsvei 4, NTNU Gloshaugen, 7491, Trondheim, Norway, Phone: +47-7359-4154, Fax: +47-73594080, e-mail: skoge@chemeng.ntnu.no. 
It is well accepted that small process gains may cause problems. For example, the requirement for avoiding input saturation is $\underline{\sigma}(G) \geq 1$, that is, a minimum gain of one is required ${ }^{1}$. This assumes that the desired output changes (setpoints) are of magnitude 1 and the allowed inputs are also of magnitude 1 , both expressed in terms of the 2-norm.

It is less clear whether large process gains pose a problem. Skogestad and Postlethwaite $^{2}$ consider the condition number, defined as $\gamma(G)=\bar{\sigma}(G) / \underline{\sigma}(G)$ and make the following statement: A large condition number may be caused by a small value of $\underline{\sigma}(G)$, which is generally undesirable. On the other hand, a large value of $\bar{\sigma}(G)$ is not necessarily a problem.

On the other hand, Moore ${ }^{3}$ claims that high sensitivity (high gains) can be a problem because of low input resolution in valves and actuators. He states: Valves and other actuators all have a minimum resolution with respect to positioning. These limitations restrict the fine adjustments often necessary for high gain processes to reach a steady operation. If the fine adjustment necessary for steady state is less than the resolution of the valve, sustained oscillations are likely to occur. Consider, for example, a steam valve with resolution of $\pm 1.0 \%$. If a valve position of $53.45 \%$ is necessary to meet the target temperature, then the valve will, at best, settle to a limit cycle that hunts over a range from about $55 \%$ to $53 \%$. If the process gain is 10, the hunting of the valve will cause a limit cycle in the control temperature of $20 \%$. In this paper, we confirm that limit cycles are unavoidable under such conditions, but we find that it is the process gain at the frequency of the limit cycles, and not at steady-state, that matters for controllability.

McAvoy and Braatz ${ }^{4}$ argue along the same lines as Moore ${ }^{3}$ and state that for control purposes the magnitude of steady-state process gain $(\bar{\sigma}(G))$ should not exceed about 50 .

In this paper two main types of input errors are discussed. We first consider the input oscillations caused by restrictions of the input (valve) resolution. Later, in section 7, we consider input (load) disturbance which is not related to the valve resolution problems. Most of the results are derived for first-order plus delay processes. When possible, more general derivations are presented.

\section{Restricted input resolution and limit cycles}

As mentioned by Moore ${ }^{3}$ and proved below, feedback control with restricted (low) input resolution results in limit cycles (hunting). A simple representation of restricted (low) input resolution is to use a quantized input as depicted in Figure 1. The output

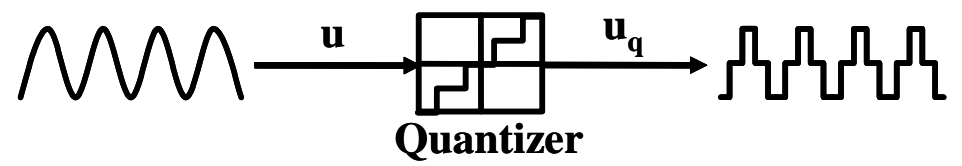

Figure 1: Quantization of a smooth signal.

$u_{q}$ from the quantizer is

$$
u_{q}=q \cdot \operatorname{round}(u / q)
$$


where $q$ is the quantization step and the round function takes its argument to the nearest integer. This may, for example, represent restricted valve resolution and to some extent valve stiction and valve dead band ${ }^{5}$. An extreme case with only one quantization step is an on-off valve.

Figure 2 shows a feedback system with a quantizer. Here $G(s)$ is the plant transfer function model, $K(s)$ the controller, $y$ the plant output with reference $r$, and $u$ the manipulated variable (for simplicity, the Laplace variable $s$ is often omitted). The low input resolution results in a stepwise input "disturbance" of magnitude $q$. and this again results in oscillations in the plant output $y(t)$ of magnitude $a$. Note that $a$ here is defined as the "total" amplitude from the bottom to the top of the oscillations.

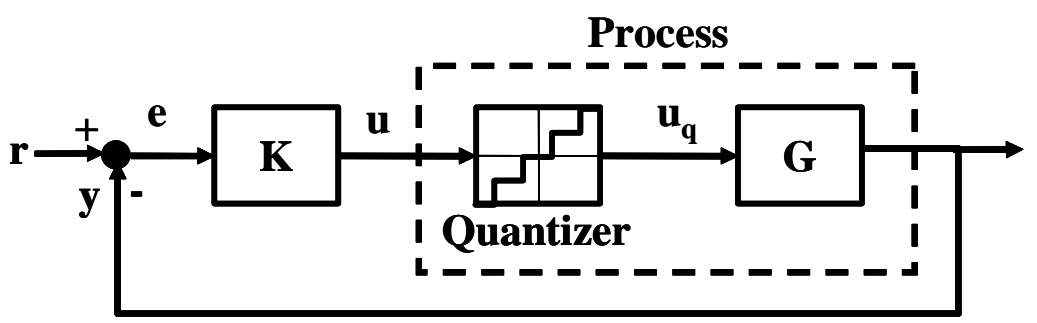

Figure 2: Feedback control of process with restricted input resolution (quantizer)

Theorem 1 For the feedback system with a quantizer in Figure 2 limit cycles are inevitable if there is integral action in the controller such that the output in average has no steady-state offset.

Proof. At steady-state the average value of the output $y$ is equal to the reference $r$, that is $y_{s s}=r$ where $y_{s s}$ denotes the average ("steady-state") value of $y(t)$ as $t \rightarrow \infty$. To achieve this the input u must on average equal the following value

$$
u_{s s}=\frac{y_{s s}}{G(0)}=\frac{r}{G(0)}
$$

where $G(0)$ denotes the steady-state plant gain. Except for the special case that $u_{s s}$ happens to exactly correspond to one of the quantizer levels $q_{i}$ (which in practice with measurement noise will not occur), the quantized input $u_{q}$ must then cycle between at least two of the quantizer levels.

Let us consider the most common case where the output cycles between the two neighboring quantizer levels to $u_{s s}$, here denoted $q_{1}$ and $q_{2}$. Let $f$ and $(1-f)$ denote the fraction of time spent at each of the two levels. Then, at steady-state (as $t \rightarrow \infty$ ) $u_{s s}=f q_{1}+(1-f) q_{2}$ and we have the following expression for the fraction of time $u$ spends at level $q_{1}$ :

$$
f=\frac{q_{2}-u_{s s}}{q_{2}-q_{1}}
$$

Note that the closer $u_{s s}$ is to one of the quantizer levels, the longer the time $u_{q}$ will remain on it.

Example 1. As an example consider the system simulated in Figure 3 where $q_{1}=0$ and $q_{2}=0.03$ (this may represent an on/off valve). The third order plant model is

$$
G(s)=\frac{100}{(10 s+1)(s+1)^{2}}
$$


and we use a PI-controller

$$
K(s)=K_{c}\left(\frac{\tau_{I} s+1}{\tau_{I} s}\right) ; \quad K_{c}=0.04, \tau_{I}=10
$$

Note that the integral time is chosen so that we cancel the dominant pole in $G(s)$ (IMC tuning rule). The steady-state plant gain is $G(0)=100$. Initially, the system is at steady-state with $u_{q}=q_{1}=0$ and $y=r=0$. We then make a step change $r=1$. The steady-state plant gain is $G(0)=100$, so to achieve $y_{s s}=1$ the required average input is $u_{s s}=1 / 100=0.01$ which is closer to $q_{1}=0$ than $q_{2}=0.03$. The fraction of time $u_{q}$ remains at $q_{1}=0$ is $f=(0.03-0.01) / 0.03=0.67$. As expected, this agrees with the simulations.
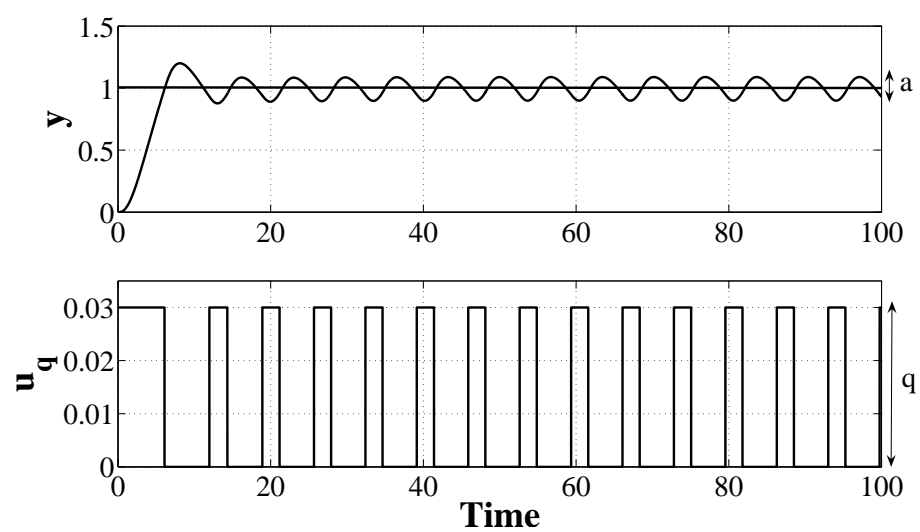

Figure 3: Simulation results for system in Example 1.

Example 2. A similar simulation example with $q_{1}=0$ and $q_{2}=0.03$ is shown in Figure 3, but for a first-order with delay plant

$$
G(s)=\frac{k e^{-\theta s}}{(\tau s+1)}
$$

with $k=100, \theta=1$ and $\tau=10$. We use the same PI-controller as in (5) with $\tau_{I}=$ $\tau=10$ and $K_{c}=0.04$. The main difference compared to Example 1 is that the step reference change is much smaller, $r=0.2$, such that the input stays a much shorter time at the upper quantizer level of $q_{2}=0.03$. The steady-state plant gain is $k=G(0)=$ 100 , so to achieve $y_{s s}=0.2$ the required average input is $u_{s s}=0.2 / 100=0.002$. From (3), the fraction of time $u_{q}$ remains at $q_{1}=0$ is $f=(0.03-0.002) / 0.03=0.93$. Again, this agrees with the simulations.

For the simulated system in Figure 3 (Example 1), the magnitude of limit cycles (oscillations) in $y$ is $a=0.189$ and the period is $T=6.72 \mathrm{~s}$. The oscillations in $y(t)$ are seen to be quite close to sinusoidal. For the simulated system in Figure 4 (Example 2 ), we have $a=0.3$ and $T=16.07 \mathrm{~s}$. However, in this case the oscillations in $y(t)$ are far from sinusoidal.

We next want to derive analytic expressions for $a$ and $T$. We first make the simplifying assumption that the resulting limit cycles are sinusoidal and then study the more general case. 

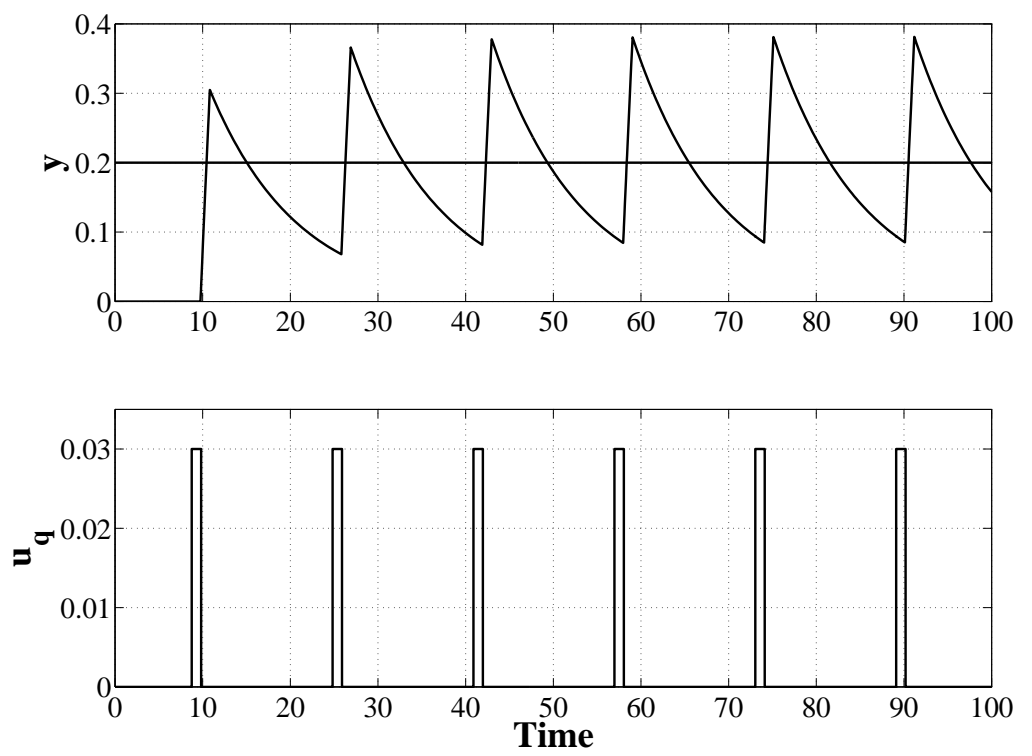

Figure 4: Simulation results for system in Example 2.

\section{Describing function analysis of oscillations (assuming sinusoids)}

The quantizer (nonlinearity) that causes the limit cycles can be regarded as a relay without hysteresis and is in the following treated as such. As an approximation, the amplitude of the oscillations can then be found analytically from an harmonic linearization or describing function analysis of the nonlinearity. This is analysis is exact if the resulting limit cycle is sinusoidal. For the feedback system in Figure 2, the condition for oscillation is given by ${ }^{7}$

$$
N\left(a_{u}\right) L(j \omega)=-1,
$$

where $N\left(a_{u}\right)$ is the describing function of the nonlinearity (quantizer) which is as a function of the amplitude $a_{u}$ of the oscillations in $u(t)$ - at the quantizer input, and $L=G K$ is the loop transfer function (excluding the quantizer). For a relay without hysteresis, the describing function is 6

$$
N\left(a_{u}\right)=\frac{4 q}{\pi a_{u}}
$$

and $q$ is the relay amplitude (quantization step). Since according to $(8), N\left(a_{u}\right)$ is a real number, it follows from (7) that $\omega$ is actually the ultimate frequency $\omega_{L, 180}$ and

$$
N\left(a_{u}\right)=\frac{1}{\left|L\left(j \omega_{L, 180}\right)\right|}=\frac{4 q}{\pi a_{u}}
$$


The amplitude of the corresponding oscillations at the plant output are $a=a_{u} /\left|K\left(j \omega_{L, 180}\right)\right|$ which leads to

$$
\begin{aligned}
a & =\frac{4 q\left|G\left(j \omega_{L, 180}\right)\right|}{\pi} \\
T & =\frac{2 \pi}{\omega_{L, 180}}
\end{aligned}
$$

where $T$ is the period of oscillation. This is exact if the limit cycles are sinusoidal.

Example 1 (continued). For the system given by (4) and (5), $\angle L\left(j \omega_{L, 180}\right)=$ $-\frac{\pi}{2}-2 \arctan \left(1 \cdot \omega_{L, 180}\right)=-\pi$ which yields $\omega_{L, 180}=1[\mathrm{rad} / \mathrm{s}]$ and $\left|G\left(j \omega_{L, 180}\right)\right|=$ 4.999. From a describing function analysis the period of oscillation is then $T=$ $\frac{2 \pi}{\omega_{L, 180}}=6.28 \mathrm{~s}$. and from (10) $a=\frac{4}{\pi} q\left|G\left(j \omega_{L, 180}\right)\right|=0.191$. This is in good agreement with the simulation results $(T=6.72 \mathrm{~s}, a=0.189)$.

First-order with delay process. Consider a first-order with delay plant $G()$ controlled by a PI-controller with $\operatorname{tau}_{I}=\tau$,

$$
\begin{aligned}
G(s) & =\frac{k e^{-\theta s}}{\tau s+1} \\
K(s) & =K_{c} \frac{\tau_{I} s+1}{\tau_{I} s}, \tau_{I}=\tau
\end{aligned}
$$

For this system we have $\angle L\left(j \omega_{L, 180}\right)=-\frac{\pi}{2}-\omega_{L, 180} \theta=-\pi$ which gives $\omega_{L, 180}=$ $\frac{\pi}{2} \frac{1}{\theta}$ and $\left|G\left(\omega_{L, 180}\right)\right|=k / \sqrt{\left(\frac{\pi}{2} \frac{\theta}{\tau}\right)^{2}+1}$. From the describing analysis in (10) and (11) we then have

$$
a=\frac{4}{\pi} \frac{q k}{\sqrt{\left(\frac{\pi}{2} \frac{\tau}{\theta}\right)^{2}+1}}: \quad T=4 \theta
$$

For small delays $(\theta / \tau \ll 1)$ this gives $a \approx \frac{8}{\pi^{2}} q \frac{k}{\tau} \theta$, and we see that amplitude of the oscillations increases proportionally with $k^{\prime}=k / \tau$ (intial slope of step response) and $\theta$. For large delays $(\theta / \tau \gg 1), a \approx \frac{4}{\pi} q k$, and we see that amplitude of the oscillations increases proportionally with $k$ (steady-state gain) and is independent of $\theta$. In all cases $a$ increases proportionally with $q$.

Example 2 (continued). With $k=100, \theta=1, \tau=10$ and $q=0.03$ (14) gives $T=4 \mathrm{~s}$ and $a=0.243$. This should be compared with the actual value from the simulations whicha are $T=16.1 \mathrm{~s}$ and $a=0.296$. Taking into account that the oscillations in $y(t)$ are far from sinusoidal, the value of $a$ in (14) obtained from the describing function analysis is quite good (about $20 \%$ too low). However, the period $T$ is a factor of four too small.

From the two examples its seems that the amplitude of $a$ in (17) from the describing function analysis is quite accurate, but that the actual period may be much larger. This conclusion is confirmed by an exact analysis for a first-order with delay plant presented next.

\section{Exact analysis of oscillations for first-order plus de- lay process}

In this section, exact results for non-sinusoidal quantized responses are derived for a first-order with delay plant controlled by a PI controller with $\tau_{I}=\tau$. The following theorem is based on the work by Wang et al. ${ }^{10}$. 
Theorem 2 For a system given by (12) and (13) set up according to the configuration of Figure 2 with quantizer level q, the amplitude and period of the limit cycle oscillations are

$$
\begin{aligned}
a & =k q \frac{1-e^{\frac{-t_{1}}{\tau}}+e^{\frac{-T}{\tau}}-e^{\frac{-\left(T-t_{1}\right)}{\tau}}}{1-e^{\frac{-T}{\tau}}} \\
T & =\theta\left(\frac{1}{1-f}+\frac{1}{f}\right)
\end{aligned}
$$

where $t_{1}=\frac{\theta}{1-f}$ and $f$ is calculated from $u_{s s}=f q_{1}+(1-f) q_{2}$.

Proof: See the appendix.

Example 2 (continued). With $f=0.933$, the amplitude and period of oscillation calculated using (15) and (16) are $a=0.2962$ and $T=16.07 \mathrm{~s}$, respectively, which matches exactly the observed results in Figure 4.

Note that the assumption $\tau_{I}=\tau$ is the reason why $a$ and $T$ are independent of the controller settings $K_{c}$ and $\tau_{I}$.

In Figure 5 the amplitude $\frac{a}{k q}$ from (15) is plotted as a function of $\frac{\theta}{\tau}$ for various values of $f$. For small delays $(\theta<<\tau), a$ increases almost proportionally $\theta / \tau$, but for large values of $\theta$ it levels off at a constant value of $a=k q$ Note that $a$ depends only weakly on $f$.

To compare, the dashed line in Figure 5 represents (14) from the describing function analysis. The agreement is generally very good with a maximum difference of $27 \%$ for large values of $\theta / \tau$.

On the other hand, note that the period of oscillation can be very different from that found with the describing function analysis. From (16) the period $T$ increases proportionally with the delay $\theta$, which agress with the value $T=4 \theta$ in (16) from the describing function analysis. However, in the exact analysis, $T$ also depends on $f$ and goes to infinity as $f$ approaches 0 or 1 . From (16), the minimum value $T=4 \theta$ is obtained when $f=0.5$, and only this limiting value agrees with the describing function analysis. This is not too surprising as the input is most close to "sinusoidal" when $f=0.5$.

\section{Controllability requirements for systems with restricted input resolution}

Consider a feedback system with restricted input resolution (quantized input) as shown in Figure 2. Assume there is integral action in the controller such that there are limit cycles (Theorem 1). Let $a_{\max }$ denote the maximum allowed amplitude of the limit cycles (oscillations) in $y$. Then, from (10) the following approximate controllability requirement applies:

$$
\left|G\left(j \omega_{L, 180}\right)\right|<\frac{\pi}{4} \frac{a_{\max }}{q},
$$

Note that this condition depends on the plant only, and more specifically on the plant gain at frequency $\omega_{L, 180}$.

Remark 1. The controllability condition (17) is approximate because it is based on a describing function analysis which is exact only for sinusoidal oscillations. Nevertheless, the results in the previous section indicates that the gain from the describing 

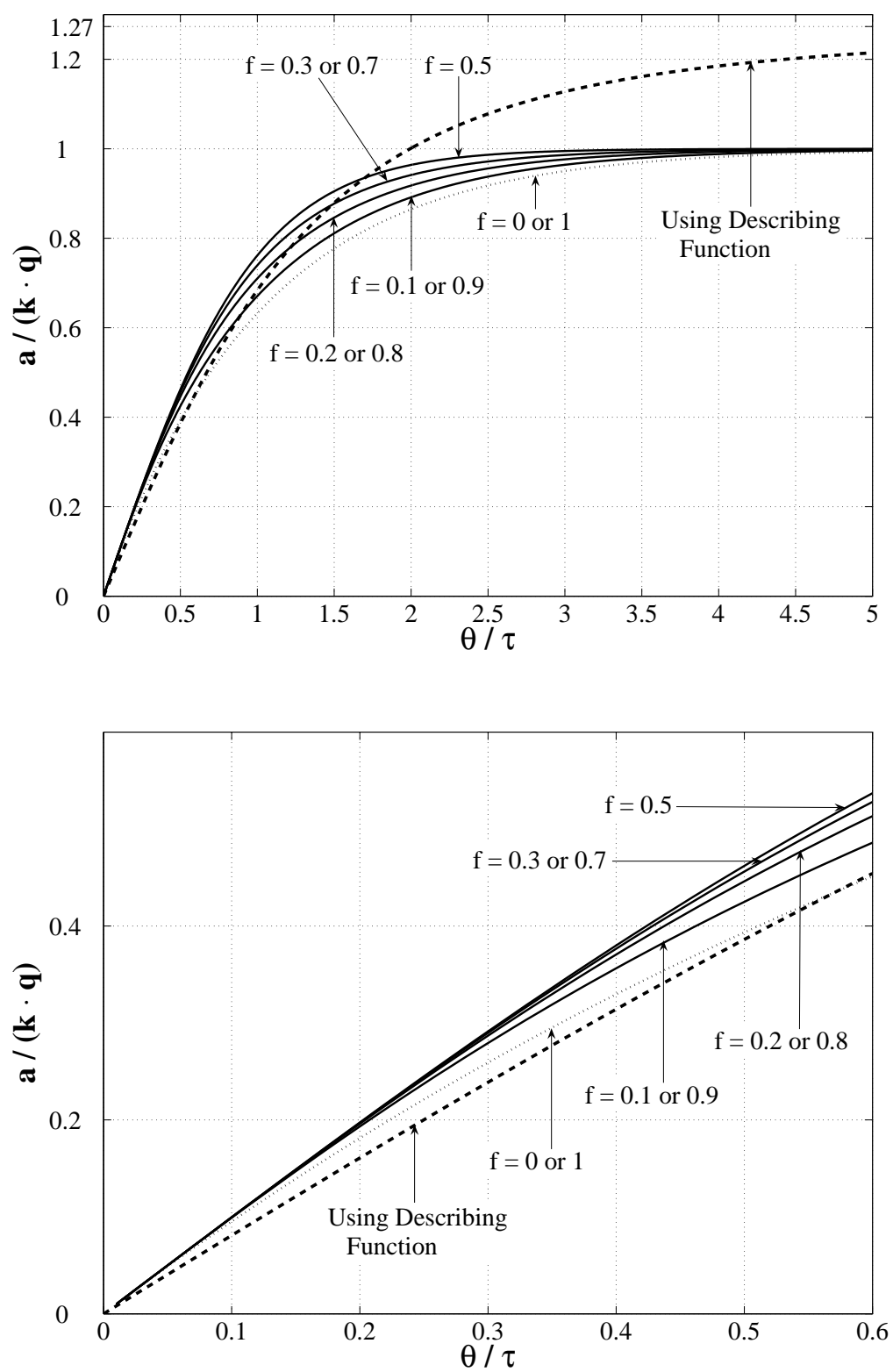

Figure 5: Amplitude $a$ in (15) plotted against $\frac{\theta}{\tau}$ for first order plus delay processes. The lower figure is a close-up of the upper figure for small values of $\frac{\theta}{\tau}$. 
function analysis is surpisingly accurate. For a first-order plus delay process, the maximum deviation was only $27 \%$ (for large values of $\theta / \tau$ ). Thus, (17) is expected to provide a tight controllability condition.

Remark 2. The controller has some effect on the condition, because $\omega_{L, 180}$ is the frequency where the sum of the phase lag in the controller $K$ and plant $G$ is $180^{\circ}$. However, for a well-tuned controller we typically have $\omega_{L, 180} \approx 1.57 / \theta$, that is, $\omega_{L}$ depends only on on the effective delay $\theta$ in the plant. Specifically, this value applies for a first (or second) order plant tuned with a SIMC PI(D)-controller ${ }^{8}$ (the value is exact when $\tau_{1}$ is smaller than about $8 \theta$ where the SIMC-rule is $\tau_{I}=\tau_{1}$, and also applies well for the case when $\tau_{1}$ is large and the SIMC-rule is $\tau_{I}=8 \theta$ ).

Remark 3. Persistent oscillations are generally undesirable. Therefore, the allowed $a_{\max }$ for oscillations is typically considerably much smaller (about 10\%) than the maximum allowed output deviation, $y_{\max }$, i.e., $a_{\max }=0.1 y_{\max }$.

\section{How to mitigate oscillations caused by restricted in- put resolution}

From the describing function analysis, the magnitude $a$ of the output oscillations for the system in Figure 2 is given by (14). The magntitude can be reduced, for example by the following means:

(a) Change the valve so that the resolution is better (smaller quantization level $q$ ).

(b) Redesign the process or the measurement devices to get a smaller effective delay $\theta$.

(c) Introduce fast, forced cycles at the input with a higher frequency than those generated "naturally". For example, one may use high-frequency pulse modulation or add a high-frequency "dither" signal (forced sinusoidal disturbance at the plant input).

(d) "Valve positioner": Use a measurement of $u_{q}$ and add a local feedback at the input to generate faster cycling, see Figure 6. This may be viewed as a combination of cases (b) and (c).

The problem with approaches (b), (c) and (d) is that fast input cycling may be undesirable, for example, because the valve cannot be moved so fast or because of excessive wear.

Frequency (pulse) width modulation. Let us consider in more detail approaches (c) and (d). A system with restricted (low) input resolution and no (average) steadystate offset is bound to cycle (Theorem 1) and the amplitude $a$ of the oscillations is given by the process gain at the frequency of oscillations, e.g. see (10). So far, we have let the system cycle at its "natural" frequency $\omega_{L, 180}$, as given by (11) and (16). However, since the gain $|G(j \omega)|$ for most processes is lower at high frequencies, an attractive alternative is make the system cycle at a higher frequency.

One approach (d) is to use a valve position controller based on measuring $u_{q}$, as shown in Figure 6. Here, the controller $K$ sets the setpoint $u_{s}$ for the valve position (input), and the "internal" valve position controller (KI) adjusts the input $u$ signal such that the actual input $u_{q}$ matches the desired input $u_{s}$ (at least on average). The valve position controller (KI) should have integral action, or a sufficiently high proportional 


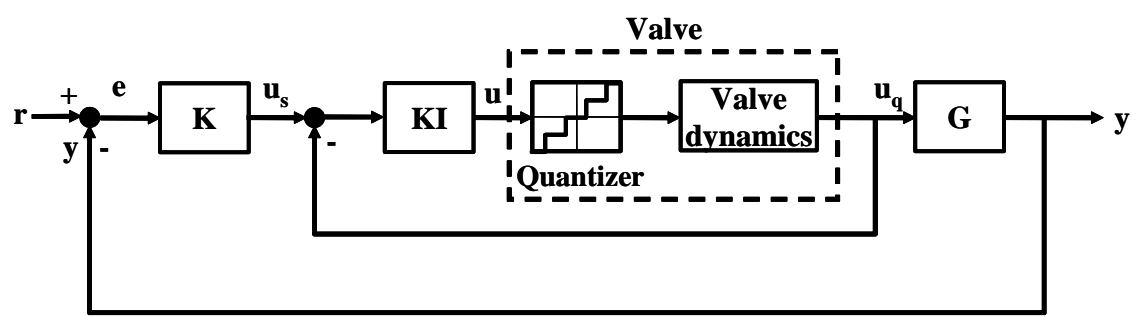

Figure 6: Frequency modulation generated using valve position controller KI.

gain, such that the internal loop cycles. The frequency of the cycling is determined by the effective delay in the "internal" valve position loop, which generally is much smaller than the delay in the overall outer loop. The results is the that the frequency of the oscillations is much higher and the resulting amplitude $a$ of the output is much smaller. This agrees with the recommendations in the Indstrument Engineers' Handbook $^{11}$, where it is noted that a positioner can reduce the dead band of a valve/actuator combination from as much as $5 \%$ to less than $0.5 \%$.

However, one may not have a measurement of the actual input $u_{q}$, and a valve position controller is in fact not necessary to reduce the effect of low input resolution. A more general approach (c) is to introduce forced pulsing by adding a frequency modulator $F$ at the output of the controller. One realization for $F$ is an internal feedback loop as depicted in Figure 7. This is similar to the valve positioner controller, except that we need an internal quantizer because there is no measurement of $u_{q}$. The modulator forces the system to cycle at a higher frequency than the one that follows "naturally". For example, forced pulsing is commonly used for on/off valves in small-scale plants where the valve may open or close every second and the controller sets the average position.

Example 3. By use of a valve position controller as shown in Figure 6, the response of the system in (12) and (13) is depicted in Figure 8. The valve dynamics is assumed to be a delay of 0.1 , and the remaining process $(G)$ has a delay of 0.9 . As it can be seen, the output amplitude is drastically reduced at the expense of high-frequency input oscillations.

P-control. Another potential approach to eliminate oscillations is to use a Pcontroller (with a sufficiently low controller gain). However, in practice this approach is not acceptable because it results in an unacceptable steady-state offset. Consider a setpoint change $r$, for which the desired input to achieve no offset is $u_{s s}=\frac{r}{G(0)}$, see (2). Assume that $r$ is such that $u_{s s}$ is in the middle between two quantization levels for the input. Then, for any non-oscillating control system, including feedforward, we have $\Delta u=\left|u_{q}-u_{s s}\right| \geq \frac{q}{2}$ and the resulting offset in the output is

$$
|y-r|=|G(0)| \cdot\left|u_{q}-u_{s s}\right| \geq|G(0)| \frac{q}{2}
$$

From this we conclude that the offset $|y-r|$ will be large for a plant with a large steady-state gain, $|G(0)|$, so P-control is in practice not recommended as a method to mitigate oscillations 


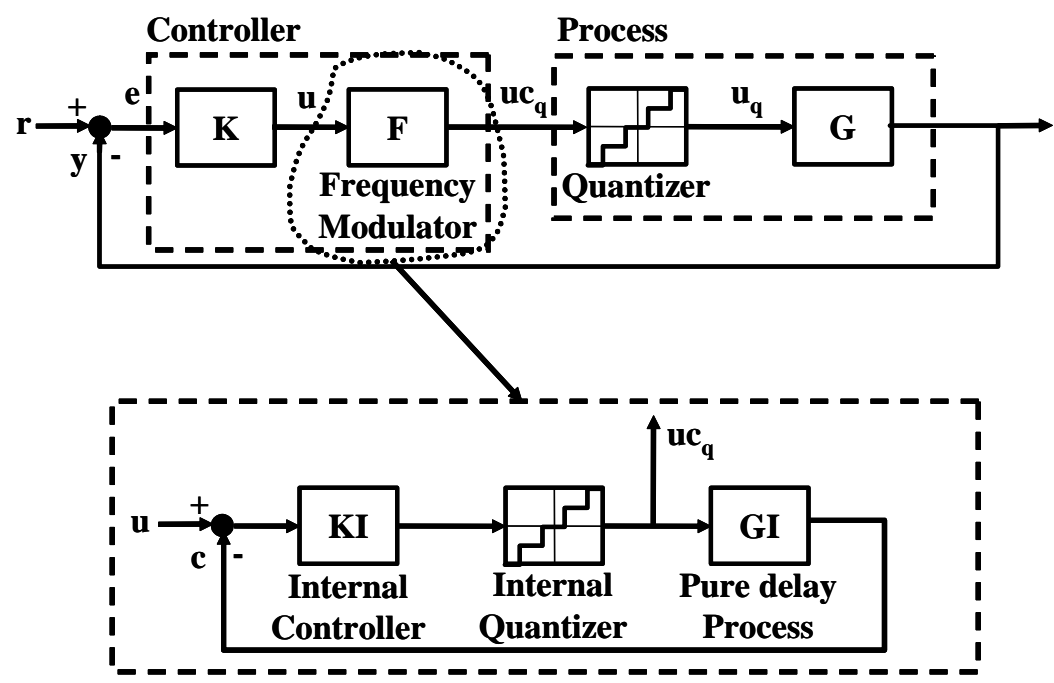

Figure 7: System with frequency modulation. The box shows one way of generating high-frequency oscillations. Alternatively, a clock may be used to set the frequency while the controller sets the pulse width.

\section{INPUT (LOAD) DISTURBANCE}

Consider a plant model in deviation variables

$$
y(s)=G(s) u(s)+G_{d}(s) d(s)
$$

where $G$ is the plant model, $G_{d}$ the disturbance model, $y$ the plant output, $u$ the manipulated variable, and $d$ the disturbance (for simplicity, the Laplace variable $s$ is often omitted). Without control the effect of disturbances on the output is $y=G_{d}(s) d$, and by "large" disturbances is meant that the product $\left|G_{d} d\right|$ is large, such that the output deviation $|y|$ will be large unnless we applyc ontrol. In this section, input disturbances are mainly considered, i.e., $G_{d}=G$. This case is illustrated in Figure 9 where $d=d_{u}$ is the disturbance at the plant input.

Feedforward control. As mentioned in the introduction, a large plant gain, especially at steady state, is a problem with feedforward control. As an example, consider a plant $y=G(u+d)$, where $d=d_{u}$ is the input (load) disturbance. Clearly, if $|G|$ is large, then $|u+d|$ needs to be small to avoid a large $|y|$. With feedforward control $u$ is adjusted based on measuring $d$. First, an accurate measurement of $d$ is required and it must be possible to adjust $u$ such that $|u-d|$ is small. The latter is not possible with restricted input resolution. For example, returning to the example of Moore ${ }^{3}$ from the introduction; $|u-d|=2 \%$ and $|G|=10$ gives $|y|=20 \%$, all at steady state.

Feedback control. On the other hand, with feedback control, "large" disturbances are not necessarily a problem, at least not at steady state. Consider a single disturbance $d$. Without control the steady-state sinusoidal response from $d$ to the output is $y(\omega)=G_{d}(j \omega) d(\omega)$, where phasor notation is used and $|d(\omega)|$ denotes the magnitude of the disturbance at frequency $\omega$. We assume that the magnitude is independent of the frequency, i.e. $|d(\omega)|=d_{0}$ and assume that the control objective is that the output 


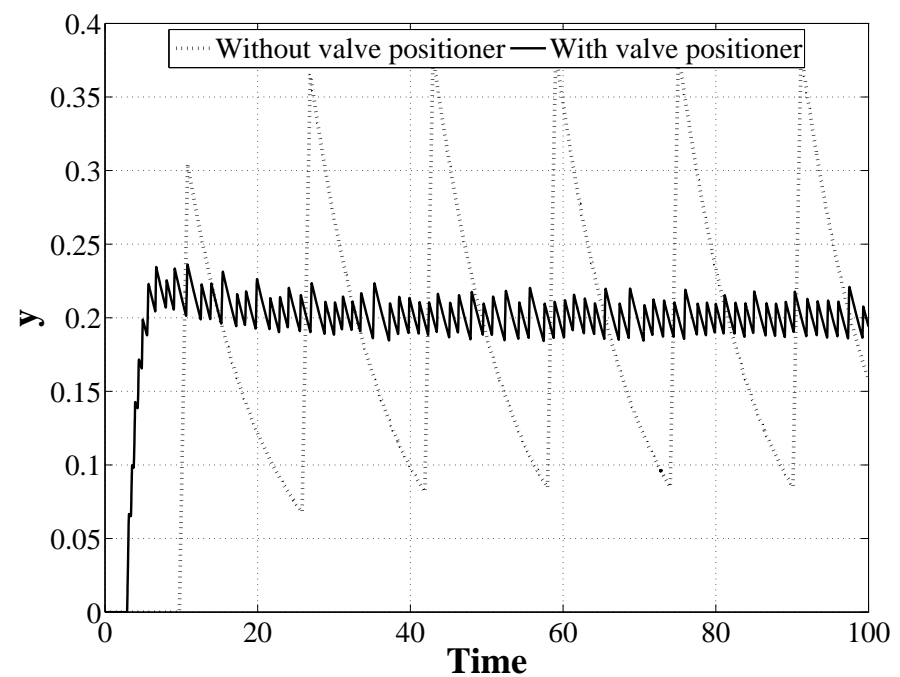

With valve positioner
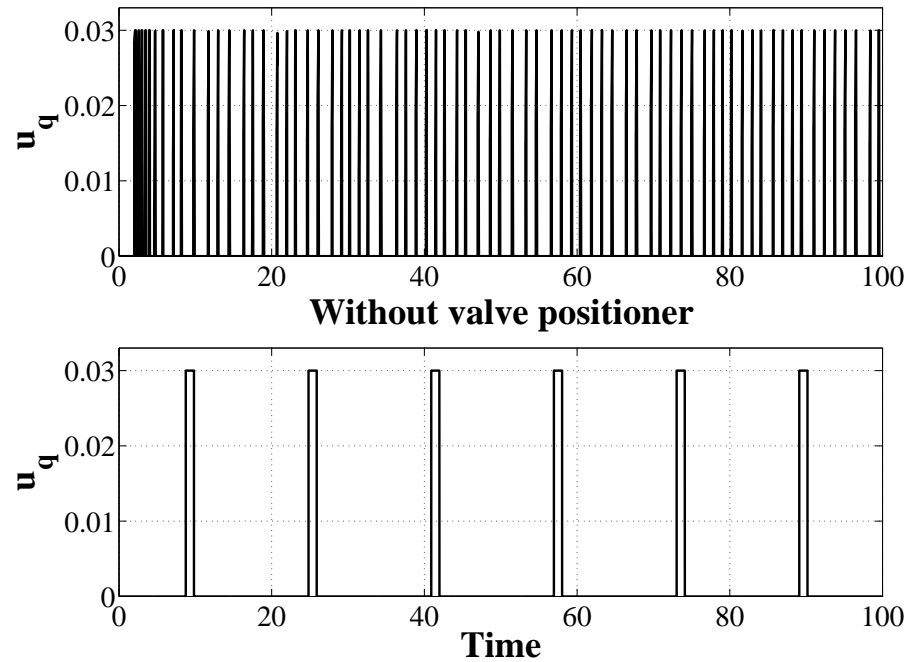

Figure 8: Effect of using valve position control for the system in Example 2. 
is less than $y_{\max }$ at any given frequency, i.e., $|y(\omega)|<y_{\max }$. From this, one can immediately draw the conclusion that no control is needed if $\left|G_{d}(j \omega) d_{0}\right|<y_{\max }$ at all frequencies (in which case the plant is said to be "self-regulating"). If $\left|G_{d}(j \omega) d_{0}\right|>$ $y_{\max }$ at some frequency, then control is needed. With feedback control $(u=-K y)$ we get $y(s)=S(s) G_{d}(s) d(s)$, where $S=(I+G K)^{-1}$ is the sensitivity function. The requirement $|y(\omega)|<y_{\max }$ then becomes

$$
|S(j \omega)| \cdot\left|G_{d}(j \omega)\right||d(\omega)|<y_{\max }, \quad \forall \omega
$$

With integral action in the controller, $|S|$ is zero at steady state, so in general it does not matter if $\left|G_{d}\right|$ is large at steady state (provided there is no problem with input saturation, but this is mainly a design rather than a control issue). However, $|S|$ increases with frequency and crosses 1 at the bandwidth frequency $\omega_{S},\left|S\left(j \omega_{S}\right)\right|=1$. At this frequency the requirement (20) gives the controllability requirement

$$
\left|G_{d}\left(j \omega_{S}\right)\right|<\frac{y_{\max }}{\left|d\left(\omega_{S}\right)\right|}
$$

Input disturbance. However, the purpose of this paper is not to consider plants for which $\left|G_{d}\right|$ is large, but rather plants for which $|G|$ is large (in practice, these are often related because all plants have disturbances at the input to the plant). To this effect, we consider input (load) disturbances $d_{u}$ for which $G_{d}(s)=G(s)$ (see Figure 9). Hence, (21) gives the following controllability bound on the allowed plant gain at frequency $\omega_{S}$

$$
\left|G\left(j \omega_{S}\right)\right|<\frac{y_{\max }}{\left|d_{u}\left(\omega_{S}\right)\right|}
$$

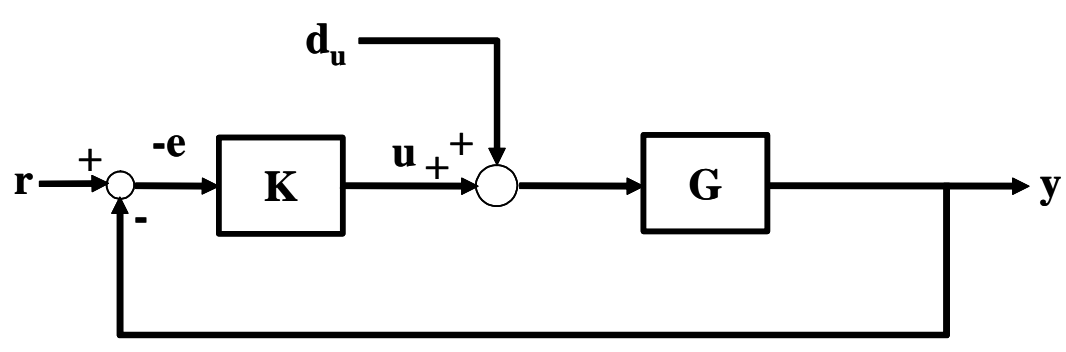

Figure 9: Block diagram of a feedback control system with disturbance at the input of the plant.

This bound is independent of the controller, and thus provides a fundamental controllability requirement. In most cases $|G|$ is smaller at high frequency, so the bound is easier to satisfy if $\omega_{S}$ is increased. However, for stability reasons the value of $\omega_{S}$ is limited, and a typical upper bound is $\omega_{S} \approx \frac{0.5}{\theta}$, where $\theta$ denotes the "effective delay" around the feedback loop ${ }^{2}$.

Input disturbances are very common, but what is the expected value of $\left|d_{u}\right|$ ? This is difficult to answer, because input disturbances have many sources. For example, in many cases the input is a valve which receives its power from a hydraulic system (e.g. the brakes of a car) or from pressured air (many process control applications). A change (disturbance) in the power system will then cause an input disturbance. The 
value of $\left|d_{u}\right|$ will vary depending on the application. If it is assumed that the system has been scaled such that the largest expected input $u$ is of magnitude 1 , then it seems reasonable that $\left|d_{u}\right|$ is at least 0.01 , and that a typical value is 0.1 or larger.

Steady-state implications. Condition (22) provides a bound on the plant gain at frequency $\omega_{S}$. The implications in terms of the steady-state are discussed next by considering a first-order with delay plant,

$$
G(s)=G_{d}(s)=\frac{k e^{-\theta s}}{(\tau s+1)},
$$

where $k=|G(0)|$ is the steady-state gain of the plant. The high-frequency asymptote is $|G(j \omega)| \approx \frac{k}{\tau \omega}=\frac{k^{\prime}}{\omega}$, where $k^{\prime}=\frac{k}{\tau}$ is the initial slope of the step response. With $\omega_{S} \approx \frac{0.5}{\theta},(22)$ gives the controllability requirement

$$
\frac{k}{\tau}=k^{\prime}<0.5 \frac{y_{\max }}{\theta\left|d_{u}\right|}
$$

(24) may seem to indicate that a plant with a large steady-state gain $k$ is fundamentally difficult to control (see case 1 below). However, as discussed in case 2 this is not always true because from (22) it is the gain at frequency $\omega_{S}$ that should be small and a process can have a large steady-state gain while having a small gain at high frequency.

Case 1. In some cases a large steady-state gain $k$ implies a large gain at high frequencies, resulting in not being able to satisfy the controllability requirement in (21). A physical example is a pH-neutralization process as studied in chapter 5 in Skogestad and Postlethwaite ${ }^{2}$. The component balance for the excess of acid $y$ gives the model $\tau_{h} s y(s)=\frac{1}{\epsilon} u(s)-y(s)$. where $\tau_{h}$ is the residence time and $u$ the neutralization flow. This is on the form of (23) with $k=1 / \epsilon$ and $\tau=\tau_{h}$. The reason for the small value of $\epsilon$ is that the desired concentration in the tank $(y)$ can be in the order of $10^{6}$ smaller than in the neutralization inflow. Because of the large high-frequency gain, this plant is not controllable according to (22), and a design change is required, for example, where the neutralization is done in several steps (tanks) rather than in a single step.

Case 2. As an example of a case where a large steady-state gain does not imply control problems, consider a near-integrating process:

$$
G(s)=\frac{k^{\prime}}{s+\epsilon} e^{-\theta s}
$$

This is on the form of (23) with $k=\frac{k^{\prime}}{\epsilon}$ and $\tau=\frac{1}{\epsilon}$. Thus, as $\epsilon \rightarrow 0$, the steady-state gain $G(0)=\frac{k^{\prime}}{\epsilon}$ approaches infinity, but the high-frequency slope of the gain $k^{\prime}$ remains finite as it is independent of $\epsilon$, so (24) may not impose any controllability limitation. A physical example is a liquid level where $\epsilon$ represents the self-regulating effect. The mass balance may be written as $s \Delta V(s)=\Delta q_{\text {in }}-\Delta q_{\text {out }}$, where the linearized outflow is $\Delta q_{\text {out }}=k^{\prime} \Delta Z(s)+\epsilon \Delta V(s)$ and $Z$ is the valve position. $\epsilon \rightarrow 0$ for the case when the outflow only depends weakly on $V$. With $y=\Delta V, u=\Delta Z$, and $d=\Delta q_{\text {in }}$, this results in a model of the form in (25) and (23).

\section{DISCUSSION}

We have derived expressions for the amplitude and period of oscillations that result with feedback control of a system with restricted input resolution (quantizer). Importantly, the amplitude and period were found (under certain assumptions about the 
integral time) to be independent of the controller gain. However, note that the time before cycling actually starts may be considerably longer than the period $T$ of the oscillations, and that this start-up time does depend on the controller gain. By detuning the controller (reducing the controller gain) it generally takes longer time for the oscillatings to start. This is confirmed by the simulations in Figure 3 in McAvoy and Braat ${ }^{4}$ where a detuned controller gives no oscillations with a simulation time of 80 s. However, it is easily confirmed that oscillations do indeed develop if the simulation time is extended to $95 \mathrm{~s}$ or more.

In this paper, we have considered the effect of input (valve) inaccuracy and input load disturbances, with the corresponding controllability requirements

$$
\begin{gathered}
\left|G\left(j \omega_{L, 180}\right)\right|<\frac{\pi}{4} \frac{a_{\max }}{q} \\
\left|G\left(j \omega_{S}\right)\right|<\frac{y_{\max }}{\left|d_{u}\left(\omega_{S}\right)\right|}
\end{gathered}
$$

Which condition is the more restrictive? There is no general answer, but let us first consider two reasons for why the latter (input disturbance) may be more restrictive. First, the input disturbance $\left|d_{u}\right|$ is normally larger than the quantization step $q$. Second, the bound for input load disturbance occurs at a lower frequency $\omega_{S}$ where the gain $|G(j \omega)|$ is generally larger than at frequency $\omega_{L, 180}$. Specially, assume that the magnitude of the first order plus delay plant in the high-frequency range can be approximated by $|G(j \omega)|=\frac{k}{\tau \omega}$. Then, taking the typical values $\omega_{S}=\frac{0.5}{\theta}$ and $\omega_{L, 180}=\frac{1.5}{\theta}$, we get

$$
\frac{\left|G\left(j \omega_{S}\right)\right|}{\mid G\left(j \omega_{L, 180} \mid\right.} \approx \frac{\omega_{L, 180}}{\omega_{S}} \approx 3
$$

This leads to the conclusion that the output deviation caused by an input disturbance is likely to be larger than the sustained output varuations caused by restricted input resolution. On the other hand, we are less likely to accept sustained oscillations $\left(a_{\max }\right)$ than short-time deviations $\left(y_{\max }\right)$, so one could argue that $a_{\max }$ is usually smaller than $y_{\max }$ (a typical value may be $a_{\max }=0.2 y_{\max }$ ). In summary, it is not clear which is the more restrictive.

McAvoy and Braatz ${ }^{4}$ state that, for control purposes, the magnitude of the steadystate process gain $(k=\bar{\sigma}(G(0)))$ should not exceed 50 . In this paper, we have derived controllability conditions, (17) and (22), that limit the plant gain at frequencies $\omega_{L, 180}$ and $\omega_{S}$, respectively. These conditions have some implications for the steady-state gain which in special cases may provide some justification for the rule-of-thumb of McAvoy and Braatz ${ }^{4}$. Specifically, the expression (18) for steady-state offset with Pcontrol gives $k \leq \frac{2|y-r|}{q}$. For example, with $q=0.02$ and $|y-r|_{\max }=a_{\max }=0.2$ this requires $k<20$. Thus, P-control should only be used for plants with a small steady-state gain. Furthermore, (22) may be rewritten as in (24) to get $k<0.5 \frac{y_{\max } \tau}{\theta\left|d_{u}\right|}$. If we select $\left|y_{\max }\right|=1,\left|d_{u}\right|=0.1$, and $\frac{\tau}{\theta}=10$ (similar to that used in the simulation in McAvoy and Braatz ${ }^{4}$ ) then we derive a bound $k<50$. However, note that the bounds (18) and (24) do not imply that large steady-state gains are always a problem for control. First, (24) is derived for a first-order with delay model where $k$ and $\tau$ are assumed independent, whereas they often are coupled, e.g. see (25). Second, (18) applies to P-control and the implication is that integral action needs to be added for control of such processes. 
In the introduction, we referred to a case by Moore ${ }^{3}$ which seems to prove that a large steady-state gain (i.e. large gain at zero frequency) gives large output variations (poor control) when we have restricted valve resolution. However, in practice the system will not cycle at a low frequency, but at a higher frequency $\left(\omega_{L, 180}\right)$ where the process gain is smaller and the resulting output variables are therefore smaller. We may also introduce forced cycling or use valve position control to further reduce the output variation.

\section{CONCLUSION}

In this paper, controllability requirements are derived for two kinds of input errors, namely (1) restricted input resolution (e.g. cased by valve inaccuracy) and (2) input disturbances.

(1) Limited input resolution with integral feedback control (no steady-state offset) causes limit cycle behavior (oscillations) (Theorem 1). The magnitude of the oscillations can be reduced by pulse modulating the input signal or using valve position control, but this assumes that frequent input movements are acceptable. The controllability requirement derived from an approximate describing function analysis, assuming no forced oscillations, is

$$
\left|G\left(j \omega_{L, 180}\right)\right|<\frac{\pi}{4} \frac{a_{\max }}{q},
$$

where $L=G K$ and, typically, $\omega_{L, 180} \approx \frac{1.5}{\theta}$ ( $\theta$ is the effective delay in the loop). $a_{\max }$ is the allowed magnitude for the resulting sustained output oscillations (limit cycles). This expression agrees well (within 27\%) with an exact nonlinear analysis for a firstorder plus delay process. With forced oscillations (pulse modulating the input signal), we can select the frequency $\omega$ to be much higher than the "natural" cycling frequency $\omega_{L, 180}$ and the controllability limitations are generally less restrictive.

(2) For input (load) disturbances of magnitude $\left|d_{u}\right|$, the controllability requirement is

$$
\left|G\left(j \omega_{S}\right)\right|<\frac{y_{\max }}{\left|d_{u}\left(\omega_{S}\right)\right|},
$$

where $y_{\max }$ is the allowed magnitude of the resulting short-term output deviation, and and typically $\omega_{S} \approx \frac{0.5}{\theta}$.

In summary, large gains at frequencies around the closed-loop bandwidth $\left(\omega_{S}, \omega_{L, 180}\right)$ may cause problems with feedback control. There is no controllability condition that involves the steady-state gain $k=|G(0)|$ only, so a large steady-state gain is not by itself a problem for feedback control.

\section{APPENDIX - Proof of Theorem 1}

Consider the first-order plus delay process in (12). Now, assume this process is excited by a periodic and persistent input (it is applied since $t>0$ ) of the form given by Figure 10. It represents the signal generated from a relay without hysteresis in which $q_{1}$ and $q_{2}$ are the limit values, $t_{1}$ is the time interval where $u_{q}$ remains in $q_{1}$, and $T=t_{1}+t_{2}$ is the period of oscillation. This signal can be represented in Laplace domain as a series of steps delayed in time. Assume now, without loss of generality that $q_{2}=0$ and $q_{1}=q$. The resulting transformed signal is given by 


$$
u_{q}(s)=\frac{q}{s}\left(1-e^{-t_{1} s}+e^{-T s}-e^{-\left(t_{1}+T\right) s}+e^{-2 T s}-e^{-\left(t_{1}+2 T\right) s}+\cdots\right)
$$

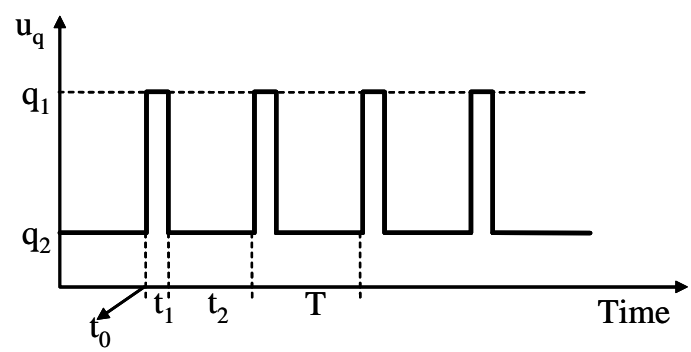

Figure 10: Input to be applied to the system in (15).

When this signal is applied to the process in (12), oscillations result in the output.

The set of maximum (or minimum) values of these oscillations are such that $t=$ $\left\{t \mid t=t_{1}+m T+\theta, \forall m \in \mathbb{N}\right\}$ and the minimum (or maximum) values are found in the set $t=\{t \mid t=m T+\theta, \forall m \in \mathbb{N}\}$.

The maximum (or minimum) at $\theta+T<t \leq \theta+t_{1}+T$ is

$$
y(s)=\frac{k}{\tau s+1} e^{-\theta s} \frac{q}{s}\left(1-e^{-t_{1} s}+e^{-T s}\right),
$$

which inverted to the time domain gives

$$
y(t)=k q\left(1-e^{-(t-\theta-T) / \tau}+e^{-\left(t-\theta-t_{1}\right) / \tau}+e^{-(t-\theta) / \tau}\right)
$$

The maximum (or minimum) is thus:

$$
y\left(t_{1}+T+\theta\right)=k q\left(1-e^{-t_{1} / \tau}+e^{-T / \tau}-e^{-\left(t_{1}+T\right) / \tau}\right)
$$

Hence, the maximum (or minimum) amplitude $y_{\text {ext } 1}$ can be extended to

$$
y_{\text {ext } 1}=k q\left(1-e^{-t_{1} / \tau}+e^{-T / \tau}-e^{-\left(t_{1}+T\right) / \tau}+e^{-2 T / \tau}-\cdots\right),
$$

which can be written as

$$
y_{\text {ext } 1}=k q\left[\left(1-e^{-t_{1} / \tau}\right)\left(1+e^{-T / \tau}+e^{-2 T / \tau}+e^{-3 T / \tau}+\cdots\right)\right]
$$

The infinite sum in (32) is given by

$$
\lim _{n \rightarrow \infty} \sum_{j=0}^{n}\left(e^{-T / \tau}\right)^{j}=\frac{1}{1-e^{-T / \tau}}
$$

where the fact that $\left(e^{-T / \tau}\right)^{n}$ converges to zero as $n$ goes to infinity is used. 
Accordingly,

$$
y_{e x t 1}=k q\left(\frac{1-e^{-t_{1} / \tau}}{1-e^{-T / \tau}}\right)
$$

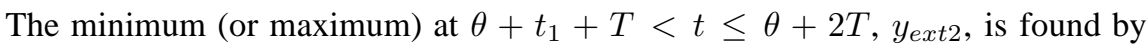
following the same development used to derive $y_{\text {ext } 1}$, i.e.

$$
y_{e x t 2}=k q\left[\frac{e^{-T / \tau}\left(1-e^{-t_{1} / \tau}\right)}{1-e^{-T / \tau}}\right],
$$

The amplitude is calculated by $a=y_{\text {ext } 1}-y_{\text {ext } 2}$ or

$$
a=k q\left(\frac{1-e^{-t_{1} / \tau}+e^{-T / \tau}-e^{-\left(T-t_{1}\right) / \tau}}{1-e^{-T / \tau}}\right)
$$

The formula in (36) depends on $t_{1}$ and $T$ which must be determined.

From Figure 2:

$$
u(s)=K(s)[r(s)-y(s)],
$$

where $K(s)$ is given by (13), $r(s)$ is a step change in reference $\left(r(s)=\frac{r_{0}}{s}\right)$, and $y(s)=K(s) G(s) u_{q}(s)$, where $G(s)$ is given by (12).

In the limit when $t \rightarrow \infty$, the quantizer behaves exactly as the relay depicted in Figure 10 and assuming that $q_{1}$ and $q_{2}$ are arbitrary values, the first three terms of $u_{q}$ are:

$$
u_{q}(s)=\frac{q_{2}}{s}+\frac{q_{1}-q_{2}}{s}\left(e^{-t_{1} s}-e^{-\left(t_{1}+t_{2}\right) s}\right),
$$

where the fact that $T=t_{1}+t_{2}$ is used.

Consider a PI-controller. Taking (38) into (37) and inverting it to time domain, the following equation for $u(t)$ in the interval $\theta \leq t<t_{0}+\theta$ is found:

$$
u(t)=\frac{K_{c}}{\tau_{I}}\left\{r_{0}\left(t+\tau_{I}\right)-k q_{2}\left[\left(\tau_{I}-\tau\right)\left(1-e^{-(t-\theta) / \tau}\right)+t-\theta\right]\right\}
$$

For the interval $\theta+t_{0} \leq t<t_{0}+t_{1}+\theta, u(t)$ is given by

$$
\begin{aligned}
u(t)= & \frac{K_{c}}{\tau_{I}}\left\{r_{0}\left(t+\tau_{I}\right)-k q_{2}\left[\left(\tau_{I}-\tau\right)\left(1-e^{-(t-\theta) / \tau}\right)+t-\theta\right]-\right. \\
& \left.k\left(q_{1}-q_{2}\right)\left[\left(\tau_{I}-\tau\right)\left(1-e^{-\left(t-t_{1}-\theta\right) / \tau}\right)+t-t_{1}-\theta\right]\right\}
\end{aligned}
$$

Likewise, for the interval $\theta+t_{0}+t_{1} \leq t<t_{0}+t_{1}+t_{2}+\theta$,

$$
\begin{aligned}
u(t)= & \frac{K_{c}}{\tau_{I}}\left\{r_{0}\left(t+\tau_{I}\right)-k q_{2}\left[\left(\tau_{I}-\tau\right)\left(1-e^{-(t-\theta) / \tau}\right)+t-\theta\right]-\right. \\
& k\left(q_{1}-q_{2}\right)\left[\left(\tau_{I}-\tau\right)\left(1-e^{-\left(t-t_{1}-\theta\right) / \tau}\right)+t-t_{1}-\theta\right]+ \\
& \left.k\left(q_{1}-q_{2}\right)\left[\left(\tau_{I}-\tau\right)\left(1-e^{-\left(t-t_{1}-t_{2}-\theta\right) / \tau}\right)+t-t_{1}-t_{2}-\theta\right]\right\}
\end{aligned}
$$


So far, no assumptions on the controller settings $\left(K_{c}\right.$ and $\left.\tau_{I}\right)$ have been made. The expressions (39)-(41) drastically simplify if the integral time is selected as $\tau_{I}=\tau$, which is an appropriate setting for many plants ${ }^{9}$.

Furthermore, for a relay without hysteresis its output $\left(u_{q}(t)\right)$ changes as its input $(u(t))$ equals to zero and since the quantizer behaves as a relay when $t \rightarrow \infty$, the following equations give relations for $t_{1}$ and $t_{2}$.

For $t=t_{0}$ :

$$
r_{0}\left(t_{0}+\tau_{I}\right)=k q_{2}\left(t_{0}-\theta\right)
$$

For $t=t_{0}+t_{1}$ :

$$
r_{0}\left(t_{0}+t_{1}+\tau_{I}\right)=k q_{2}\left(t_{0}+t_{1}-\theta\right)-k\left(q_{1}-q_{2}\right)\left(t_{0}-\theta\right)
$$

For $t=t_{0}+t_{1}+t_{2}$ :

$$
\begin{aligned}
r_{0}\left(t_{0}+t_{1}+t_{2}+\tau_{I}\right)= & k q_{2}\left(t_{0}+t_{1}+t_{2}-\theta\right)-k\left(q_{1}-q_{2}\right)\left(t_{0}+t_{2}-\theta\right)+ \\
& k\left(q_{1}-q_{2}\right)\left(t_{0}-\theta\right)
\end{aligned}
$$

Combining (42)-(44) the following expressions give the period $T$ of the oscillations:

$$
\begin{aligned}
t_{1} & =\frac{k\left(q_{1}-q_{2}\right) \theta}{k q_{1}-r_{0}} \\
t_{2} & =\frac{k\left(q_{1}-q_{2}\right) \theta}{r_{0}-k q_{2}} \\
T & =t_{1}+t_{2}
\end{aligned}
$$

On average, the input must equal the steady-state value $u_{s s}=\frac{y_{s s}}{G(0)}=\frac{r_{0}}{k}$ (where $k=G(0)$ ), and if this does not happen to exactly correspond to one of the quantizer level, the quantized input $u_{q}$ will cycle between the two neighboring quantizer levels, $q_{1}$ and $q_{2}$. Let $f$ and $(1-f)$ denote the fraction of time spent at each level. Then, at steady state $u_{s s}=\frac{r_{0}}{k}=f q_{1}+(1-f) q_{2}$ and from this expression $f$ is found to be

$$
f=\frac{r_{0}-k q_{2}}{k\left(q_{1}-q_{2}\right)}
$$

From (48),

$$
\begin{aligned}
t_{1} & =\frac{\theta}{1-f} \\
T & =\theta\left(\frac{1}{1-f}+\frac{1}{f}\right),
\end{aligned}
$$

which completes the proof. 


\section{References}

(1) Morari, M. Design of Resilient Processing Plants III - A General Framework for the Assessment of Dynamic Resilience. Chemical Engineering Science, 1983, 38, 1881.

(2) Skogestad, S.; Postlethwaite, I. Multivariable Feedback Control: Analysis and Design; 2nd Edition, John Wiley \& Sons: Chichester, UK, 2005.

(3) Moore, C. F. Selection of Controlled and Manipulated Variables; In Practical Distillation Control, Van Nostrand Reinhold: New York, 1992.

(4) McAvoy, T. J.; Braatz, R. D. Controllability of Process With Large Singular Values. Ind. Eng. Chem. Res., 2003, 42, 6155.

(5) Shoukat Choudhory, M.A.A.; Thornhill, N.F.; ShaH N.L. Modelling valve stiction. Control Engineering Practice, 2005, 13, 641.

(6) Slotine, J. E.; Li, W. Applied Nonlinear Control; Prentice-Hall International Editions: New Jersey, 1991.

(7) Aström, K. J.; Hägglund, T. Automatic Tuning of PID Controllers; Instrument Society of America: USA, 1988.

(8) Skogestad, S. Simple Analytic Rules for Model Reduction and PID Controller Tuning. Journal of Process Control, 2003, 13, 291.

(9) Smith, C. L.; Corripio, A. B.; Martin, J. Jr. Controller Tuning from Simple Process Models. Instrumentation Technology, 1975, 39.

(10) Wang, Q.; Hang, C.; Zou, B. Low-Order Modelling from Relay Feedback. Process Design and Control, 1997, 36, 375.

(11) B.G Liptak. Instrument Engineers' Handbook. Volume II: Process control and optimization. CRC Press, 4th edition, 2006

\section{LIST OF CAPTIONS}

- Figure 1: Quantization of a smooth signal.

- Figure 2: Feedback control of process with restricted input resolution (quantizer).

- Figure 3: Simulation results for system in Example 1.

- Figure 4: Simulation results for system in Example 2.

- Figure 5: Amplitude $a$ in (15) plotted against $\frac{\theta}{\tau}$ for first order plus delay processes. The lower figure is a close-up of the upper figure for small values of $\frac{\theta}{\tau}$.

- Figure 6: Frequency modulation generated using valve position controller KI. 
- Figure 7: System with frequency modulation. The box shows one way of generating high-frequency oscillations. Alternatively, for an on/off valve a clock may be used to set the frequency while the controller sets the pulse width.

- Figure 8: Effect of using valve position control for the system in Example 2.

- Figure 9: Block diagram of a feedback control system with disturbance at the input of the plant.

- Figure 10: Input to be applied to the system in (15). 